\title{
La formación del compromiso ético en investigación educativa: resultados de una experiencia de innovación docente en educación superior
}

\section{The development of students' ethic commitment in educational research: An innovative teaching experience in higher education}

Laura Corbella Molina

Universitat Autónoma de Barcelona, Barcelona, España

laura.corbella@uab.cat

https://orcid.org/0000-0002-2151-089X

Clara Sanz Escutia

Universitat Autónoma de Barcelona, Barcelona, España

clara.sanz@uab.cat

https://orcid.org/0000-0001-8945-660X

Laura Arnau Sabates

Universitat Autónoma de Barcelona, Barcelona, España

laura.arnau@uab.cat

(iD https://orcid.org/0000-0003-3359-4071

Recepción: 01/06/2021 | Aceptación: 30/07/2021 | Publicación: 10/09/2021

Cómo citar (APA, séptima edición):

Corbella Molina, L., Sanz Escutia, C., y Arnau Sabates, L. (2021). La formación del compromiso ético en investigación educativa: resultados de una experiencia de innovación docente en educación superior. INNOVA Research Journal, 6(3), 135-154. https://doi.org/10.33890/innova.v6.n3.2021.1772

\footnotetext{
Resumen

La formación inicial de los investigadores en educación es determinante para garantizar la adquisición de conocimientos y de técnicas que contribuyan a la calidad de la investigación, y también para el desarrollo de su competencia ética. Este trabajo presenta los resultados de la aplicación de una innovación educativa para mejorar la competencia ética en una asignatura de 
investigación educativa. El objetivo es evaluar la adquisición de dicha competencia en un grupo de 80 estudiantes de los grados de Pedagogía y Educación Social. Para ello, a través de un enfoque cuantitativo, se llevó a cabo un diseño pre-experimental pretest-postest. El instrumento de recogida de información fue un cuestionario en el que se exponían ciertos dilemas éticos comunes en los procesos de investigación. Para analizar los resultados cuantitativos se usó la prueba T-test, y para las respuestas abiertas se llevó a cabo un análisis de contenido siguiendo un proceso de categorización deductivo-inductivo. El estudio pone de manifiesto que el proceso de innovación docente ha contribuido a desarrollar y mejorar la competencia ética en la investigación educativa, aunque el discurso de los estudiantes tiende a ser muy normativo, justificando el carácter ético de sus decisiones apelando a protocolos y/o normas. Surge la necesidad de fomentar más la reflexión crítica y la responsabilidad individual. Se concluye que el desarrollo de la competencia ética en la investigación educativa no solamente necesita el compromiso de los docentes y del alumnado, sino también de las instituciones de educación superior.

Palabras claves: investigación educativa; educación ética; formación de investigadores; educación superior; innovación docente.

\begin{abstract}
The initial training of educational researchers is crucial to ensure the acquisition of knowledge and techniques that contribute to the quality of research, and also to the development of their ethical competence. This paper presents the results of the application of an educational innovation in the classroom to improve student's ethical competence in an educational research course. The aim is to evaluate the acquisition of this competence in a group of 80 students of the degrees of Pedagogy and Social Education. To this end, a pre-experimental pretest-posttest design was carried out using a quantitative approach. The instrument used to collect information was a questionnaire in which certain ethical dilemmas common to research processes were presented. A t-test was used to analyse the quantitative results, and for the open-ended responses a content analysis was carried out following a deductive-inductive categorisation process. The study shows that the teaching innovation process has contributed to the development and improvement of ethical competence in educational research, although the students' discourse tends to be very normative, justifying the ethical nature of their decisions by appealing to protocols and/or norms. There is a need to encourage more critical reflection and individual responsibility. It is concluded that the development of ethical competence in educational research needs not only the commitment of teachers and students, but also of higher education institutions.
\end{abstract}

Keywords: educational research; ethics education; research training; higher education; teaching innovation.

\title{
Introducción
}

\section{La ética en la investigación educativa}

La investigación educativa no es solo investigar en educación. Tiene que ver con investigar para cambiar, mejorar y fundamentar la práctica educativa (Brown, 2019). En definitiva, tiene como objetivo mejorar la práctica y los procesos educativos, así como la vida de las personas. La 
La formación del compromiso ético en investigación educativa: resultados de una experiencia de innovación docente en educación superior.

investigación educativa trabaja con y para las personas y, por su carácter político-ético, transformador, cuestionador e innovador, está caracterizada por un gran componente ético-moral (American Psychological Association, 2017; Buendía y Berrocal, 2001).

El fin de la investigación científica pasa por ponerse en relación con el entorno para conocerlo y comprenderlo mejor. Dentro del ámbito científico en general y específicamente en los estudios con las personas, cualquier proceso de investigación tiene un impacto en los agentes, las instituciones y los procesos involucrados. Se genera, de esta manera, una responsabilidad que marca el ethos investigador. Además, corrientes posestructuralistas afirman que la ética y la educación radican en la relación con el otro dada su condición de vulnerabilidad (Ortega, 2020). Por este motivo, se hace necesaria la adquisición de un compromiso ético, para garantizar la dignidad y los derechos de las personas que participan en la investigación y la búsqueda, a su vez, de una sociedad justa y democrática (Bridges, 2018).

En cualquier ámbito de la investigación se presentan numerosas cuestiones éticas en la toma de decisiones de la persona investigadora. Existen ciertos dilemas comunes en todo proceso y algunos específicos de la investigación educativa. Diferentes autores han analizado los problemas y dilemas éticos de esta área (Brown, 2019; Buendía y Berrocal, 2001; Espinoza y Calva, 2020; Paz, 2018; Sánchez, 1997). Estos dilemas están relacionados con los ideales de la investigación, sus propósitos y con los escenarios educativos en los que se desarrolla. La American Psycologist Asociation (2017), APA en adelante, es un referente en este aspecto dentro del ámbito educativo a nivel español. APA establece cinco principios éticos para garantizar tanto la calidad como el compromiso ético con la investigación, los cuales aglutinan las responsabilidades éticas expuestas en este apartado. El contenido de estos principios se resume en tres grandes propósitos: a) garantizar el rigor de la investigación, b) garantizar el respeto por la propiedad intelectual, y c) proteger los derechos y el bienestar de los participantes.

Por todo lo expuesto, la competencia ética es de vital importancia en la investigación educativa. No solo permite realizar un mejor abordaje de los dilemas éticos presentes en el proceso de investigación, sino que además conduce a resultados más fiables (Espinoza y Calva, 2020), mejora la toma de decisiones (Zgaga, 2020) y promueve la asunción de valores responsables que forma la identidad de los y las profesionales que investigan en educación (Colina, 2011).

\section{La competencia ética en la formación inicial de investigadores educativos}

Un factor determinante para el desarrollo de la competencia ética de los investigadores educativos es su formación inicial (Colina, 2011; Espinoza y Calva, 2020; Paz, 2018; Raykov, 2020). Dentro de la educación superior, no solo se debe garantizar la adquisición de conocimientos y técnicas adecuadas para asegurar la calidad de la investigación, sino que se debe destacar de igual manera la competencia ética (Espinoza y Calva, 2020; García, 2006). Se afirma que en las carreras universitarias del campo de la educación surge la necesidad de formar profesionales críticos, investigadores y reflexivos (Riberas et al., 2014). Por el contrario, el paso por la Esta obra se comparte bajo la licencia Creative Common Atribución-No Comercial 4.0 International (CC BY-NC 4.0) 
universidad, per se, no garantiza el desarrollo de dicha competencia (Tey et al., 2014). Se hace necesario, por tanto, desarrollar practicas docentes focalizadas a alcanzar este objetivo.

Aunque hay una clara tendencia política a incorporar la competencia ética en la educación superior, sobre todo a partir de la creación del Espacio Europeo de Educación Superior (EEES), su incorporación no se materializa en prácticas docentes de aula, quedando de esta manera como una asignatura pendiente para las universidades (Chávez y Benavides, 2011; López, 2013). En investigación educativa específicamente, la formación de la competencia ética es necesaria para desarrollar la capacidad de reflexionar y tomar decisiones éticas fundamentadas, cuando se enfrenten a los problemas derivados de un proceso de investigación.

Una de las estrategias para ayudar a los estudiantes en la formación del compromiso ético es hacer esta ética explícita, infusionarla en los contenidos curriculares, y entenderla como algo inherente en la propia disciplina más que como algo adicional o sobrepuesto (Healey, 2014). En este sentido, Opazo (2011) propone como práctica docente de gran relevancia para la formación en ética en la educación superior la discusión de dilemas éticos relacionados con la investigación educativa en las aulas universitarias. Algunas experiencias investigativas demuestran su utilidad (de Jager, 2012; Díaz-Barriga et al., 2016; McLeod-Sordjan, 2014). Esta práctica docente permite no sólo darle un enfoque más competencial a las asignaturas sino que también facilita desarrollar la reflexión crítica y comprometida a través de un proceso reflexivo y dialógico, por lo que el trabajo grupal y la participación en el aula son aspectos clave en esta discusión (González, 2004).

Vemos pues que es posible aprender y mejorar la competencia ética en el estudiantado dentro de la educación superior; especialmente, en los primeros niveles o grados de consecución del modelo de Dreyfus y Dreyfus (2004), basados en la adquisición de conocimientos para la resolución de casos o situaciones. Por tanto, la formación superior, al ser en muchas ocasiones dónde se da lugar el primer contacto con un proceso de investigación, se centrará sobre todo en el desarrollo de los primeros niveles dando las herramientas para que el estudiantado pueda desarrollar y transferir durante su práctica profesional los niveles más avanzados de competencia ética.

El presente trabajo expone los resultados obtenidos en un proceso de innovación docente que tiene como propósito desarrollar la competencia ética del estudiantado de segundo curso de los grados de Pedagogía y Educación Social. El objetivo es evaluar si existe una evolución en el desarrollo de la competencia ética a través de la incorporación explícita en la formación del alumnado de contenidos sobre ética y la práctica del análisis reflexivo con diferentes dilemas éticos.

\section{Metodología}

Desde un enfoque cuantitativo, se realiza un diseño pre-experimental pretest-postest aplicado en el núcleo de una acción formativa, concretamente en el marco de la asignatura 
La formación del compromiso ético en investigación educativa: resultados de una experiencia de innovación docente en educación superior.

Investigar en Educación. Dicha asignatura consta de 12 créditos ECTS y es de carácter obligatorio en el currículo formativo de segundo curso de los grados de Educación Social y Pedagogía.

Durante el transcurso de la asignatura, se han incorporado contenidos sobre ética de manera explícita por parte del profesorado responsable, siguiendo las recomendaciones de Healey (2014) y Opazo (2011). Para ello, se incorporaron cuatro acciones docentes: incorporación transversal de la perspectiva ética en el proceso de investigación en las clases magistrales, incorporación específica de un seminario práctico específico para analizar dilemas éticos, incorporación de metodologías de aula que permitan la reflexión crítica (debates, clases invertidas...) e incorporación de trabajo autónomo por parte del estudiantado incentivándoles a trabajar la reflexión crítica.

\section{Instrumento}

Para evaluar los resultados de aprendizaje antes y después de la incorporación de la innovación docente en el currículo y el aula, se diseñó un instrumento ad hoc, siguiendo el proceso metodológico de Artino (2007). Artino (2007) presenta una herramienta para evaluar la competencia ética a través de dilemas éticos en la investigación educativa. Se trata de diferentes viñetas que presentan una situación en la que el investigador o investigadora toma una decisión y los estudiantes deben indicar que tan ética es la decisión que toman. Esta herramienta es de gran utilidad tanto para presentar los dilemas éticos a discutir en el aula como para evaluar el desarrollo de la competencia ética del estudiantado.

En el instrumento se plantean 8 dilemas éticos frecuentes en el desarrollo de cualquier trabajo de investigación educativa. Para el diseño de los dilemas se tomaron como referencia las pautas éticas que establece APA (2017) organizadas en tres grandes ejes:

Garantizar el rigor de la investigación. Relacionado con la veracidad de los datos y una actitud honesta, comprometida y transparente por parte de la persona investigadora.

Proteger los derechos y el bienestar de los participantes. Relacionado con la necesidad de informar y dar cuenta del rol del participante tanto en aspectos formales (consentimiento informado, devolución de los resultados, anonimato...) como en aspectos relacionales (idioma en el que nos dirigimos a los participantes, actitud de la persona investigadora, disponibilidad, atención a las particularidades de la persona...)

Respeto por la propiedad intelectual. Relacionado con el reconocimiento del trabajo realizado tanto por otros investigadores como por los integrantes del equipo de investigación o la honestidad sobre el propio trabajo (plagio y autoplagio).

El instrumento se compone de 8 situaciones dilemáticas, unas correctas y otras incorrectas desde un punto de vista ético (ver tabla 1). Los estudiantes tienen que graduar cada situación en una escala del 1 al 6, según si la decisión o resolución de la situación es poco ética o muy ética. Esta obra se comparte bajo la licencia Creative Common Atribución-No Comercial 4.0 International (CC BY-NC 4.0) 
Además, se incluye un espacio abierto para justificar la respuesta final de cada dilema o situación. Para validar el instrumento y constatar la variabilidad de las respuestas, se realizó un pilotaje con 5 estudiantes de otros cursos de la misma facultad.

\section{Tabla 1}

Relación de dilemas éticos del cuestionario y ejes APA

\begin{tabular}{ll}
\hline Ejes & Dilemas \\
\hline Garantizar el rigor & Dilema ético 2. Posición paradigmática \\
& Dilema ético 5. Omisión o falsificación de datos \\
& Dilema ético 8. Conservación de los datos \\
\hline Proteger los derechos de & Dilema ético 1. Elección del tema \\
los participantes & Dilema ético 4. Acceso a la muestra \\
& Dilema ético 6. Retorno a los participantes \\
\hline Propiedad intelectual & Dilema ético 3. Citación de las fuentes \\
& Dilema ético 7. Autoplagio \\
\hline
\end{tabular}

Fuente: elaboración propia.

\section{Muestra}

La muestra del estudio está formada por 80 alumnos de la asignatura obligatoria Investigar en Educación del segundo curso de los grados de Pedagogía y Educación Social de la Universidad Autónoma de Barcelona. La participación fue voluntaria y se planteó como una tarea complementaria en el marco de la asignatura.

Del total de participantes, el 86,2 \% eran mujeres. Pese a no tratarse de una muestra aleatoria, el porcentaje de mujeres es representativo del perfil del estudiantado de los dos grados implicados (Agència per a la Qualitat del Sistema Universitari de Catalunya, 2014). El 61.3\% de los participantes había tenido un contacto previo con el ámbito de la investigación, entendiendo como contacto previo la realización de otros trabajos de la universidad, el trabajo de investigación del bachillerato o la participación en otros proyectos fuera del contexto de educación formal.

\section{Plan de análisis}

Para describir el perfil de los participantes se ha utilizado la estadística descriptiva univariada. Para analizar los contrastes pre-postest se ha utilizado la comparación de medias mediante la prueba $\mathrm{T}$ de Student para muestras relacionadas, y se aplicado, como medida de comprobación, su versión no paramétrica (T-Wilcoxon). Para el análisis de las respuestas abiertas se ha utilizado la técnica de análisis de contenido, siguiendo un proceso de categorización deductivo-inductivo con el software Nvivo. Los elementos analizados corresponden a: 1) la justificación que da el estudiantado sobre cómo aborda el dilema ético, teniendo en cuenta si tiene la capacidad de reflexionar más allá de lo normativo, basándonos en la aportación de Zgaga (2020), 
2) las habilidades que el alumnado pone en práctica, basándonos en el modelo de adquisición de competencia de Dreyfus y Dreyfus (2004), y el posicionamiento del alumnado para distinguir el tipo de dilema que se le presenta, utilizando la clasificación de Canimas (2019). El resumen de las categorías de análisis utilizadas se encuentra en la figura 1.

\section{Figura 1}

Categorías de análisis utilizadas

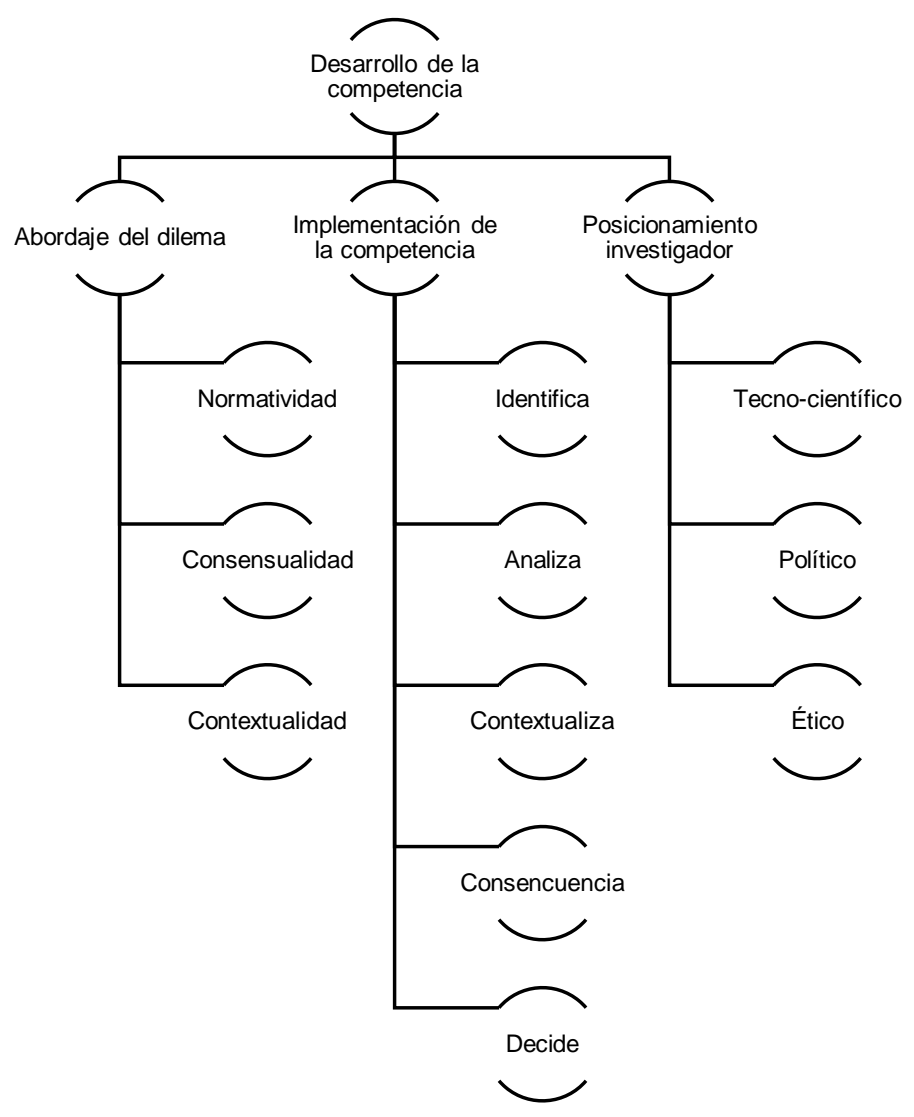

\section{Resultados}

A continuación, se presentan los resultados de la experimentación. En un primer apartado se describen los resultados de los contrastes pre-postest. En un segundo apartado se describen los resultados cualitativos y se contrastan con las medias obtenidas en el pre-postest.

\section{Resultados de los contrastes pre-postest}

Los resultados muestran que hubo mejoras estadísticamente significativas entre el pretest y el postest en todos los dilemas excepto el 2, el 4 y el 7 (ver tabla 2). Como medida de 
comprobación se ha aplicado la prueba no paramétrica de Wilcoxon y se han obtenido iguales resultados a nivel de significación. A su vez, no hay diferencias estadísticamente significativas en el pretest en función de si los participantes habían tenido contacto previo con la investigación.

\section{Tabla 2}

Resultados de los contrastes pre-postest

\begin{tabular}{lllll}
\hline & $\begin{array}{l}\text { Pre } \\
\mathbf{n = 8 0} \\
\text { Mean (SD) }\end{array}$ & $\begin{array}{l}\text { Post } \\
\mathbf{n = 8 0} \\
\text { Mean }(\text { SD) }\end{array}$ & Dif. & $\begin{array}{l}\text { T } \\
\text { (gl) }\end{array}$ \\
\hline Dilema 1 & $3.37(1.59)$ & $2.85(1.68)$ & 0.52 & $2.65(72)^{*}$ \\
\hline Dilema 2 & $3.33(1.26)$ & $3.32(1,43)$ & 0.01 & $0.06(75)$ \\
\hline Dilema 3 & $1.43(0.74)$ & $1.05(0.27)$ & 0.38 & $4.36(79)^{* * *}$ \\
\hline Dilema 4 & $2.59(1.21)$ & $2.3(1.27)$ & 0.29 & $1.71(73)$ \\
\hline Dilema 5 & $1.46(0.67)$ & $1.13(0.40)$ & 0.33 & $4.91(79)^{* * *}$ \\
\hline Dilema 6 & $4.78(1.28)$ & $5.34(1.07)$ & 0.56 & $-3.36(78)^{* *}$ \\
\hline Dilema 7 & $3.79(1.46)$ & $3.57(1.61)$ & 0.22 & $1.36(75)$ \\
\hline Dilema 8 & $3.95(1.58)$ & $4.75(1.67)$ & -0.8 & $-4.38(76)^{* * *}$ \\
\hline
\end{tabular}

Fuente: elaboración propia

$* \mathrm{p}<0,05$

$* * \mathrm{p}<0,005$

$* * * \mathrm{p}<0,0005$

\section{Resultados de las respuestas cualitativas}

En este apartado se muestran los resultados estadísticos de las respuestas dadas por los estudiantes en el pretest y el postest. Seguidamente, se presentan los resultados del análisis cualitativo de las respuestas dadas por los estudiantes ordenados por los ejes propuestos por APA (2017).

\section{Garantizar el rigor científico}

\section{Dilema 2: Posición paradigmática}

Ante el dilema planteado sobre la honestidad en la posición paradigmática de la investigación, los estudiantes consideran la respuesta a este dilema algo ética tanto en el pretest como en el postest y no existen diferencias significativas después de la aplicación de la innovación en el aula. El 50\% de estudiantes encuestados, en el pretest, utilizan una justificación científicotécnica sobre la posición paradigmática. Se centran en las cuestiones metodológicas, organizativas y prácticas y no lo vinculan con las implicaciones éticas que puede tener el hecho de partir de 
La formación del compromiso ético en investigación educativa: resultados de una experiencia de innovación docente en educación superior.

posiciones paradigmáticas preestablecidas sin tener en consideración la naturaleza del problema. En el 30\% de los casos, impera el discurso basado en el consenso, ya que lo identifican como ético porque es una decisión sometida a deliberación y aprobada por el grupo. Los que lo ven como una decisión poco ética, en un 7,5\% de los casos, hacen referencia al trato directo con los participantes del estudio, es decir, a cómo puede afectar a la persona investigada el hecho de aplicar un tipo de método u otro. En escasas ocasiones (el 11,25\%), identifican el problema ético que se presenta, pero no identifican por qué no es ético, si bien entienden que puede limitar la información recogida y que han escogido la elección fácil. Solo uno de los estudiantes reconoce que el interés propio no puede priorizarse por encima de las necesidades y del problema de la investigación. En el discurso del postest se observan algunos cambios, aunque no significativos. El discurso técnico sigue siendo elevado, dándose en el 38,75\% de los casos, y se utiliza el criterio de consenso como justificación en la misma proporción de casos que en el pretest, el 30\%. Aquellos que lo consideraban poco ético y lo relacionaban con el trato directo con los participantes, se reduce al 3,75\%. Hay un ligero aumento de las respuestas que identifican el problema ético, pasando del 11,25\% al 25\% de los encuestados, algunos de ellos incluso ofrecen alternativas. En estos casos, el discurso pasa de ser técnico a normativo, ya que identifican el problema y saben que no se debe actuar así, pero no identifican el porqué.

\section{Dilema 5: Omisión o falsificación de datos}

Ante el dilema planteado sobre la omisión y falsificación de datos en una investigación, los estudiantes consideran de media en el pretest $(\mathrm{M}=1.46 ; \mathrm{SD}=0.67)$ que la respuesta es nada ética y le otorgan puntuaciones aún más bajas en el postest $(\mathrm{M}=1.13 ; \mathrm{SD}=0.40)$, siendo la diferencia prepostest estadísticamente significativa $[\mathrm{t}(79)=4.91 ; \mathrm{p}<0.0005]$. Desde el inicio del curso, el 87,5\% de los encuestados tienen claro que la decisión adoptada de falsificar los datos del estudio no es aceptable y que se considera un engaño que invalida totalmente la investigación. El 11,25\% se muestra comprensible a la situación y, aunque no la encuentra éticamente aceptable, la podría tolerar. En el postest, los discursos siguen la misma línea, ya que un 98,75\% de los estudiantes lo consideran una decisión no aceptable. Estos discursos son aún más tajantes en afirmar que es una situación nada ética e, incluso, desaparecen las justificaciones que se daban en el pretest.

\section{Dilema 8: Conservación de los datos}

Ante el dilema planteado sobre la conservación y almacenamiento de los datos, existe una diferencia significativa entre las dos pruebas; los estudiantes consideran de media en el pretest que la actuación es algo ética y en el postest pasan a puntuarla como medianamente ética $[\mathrm{M}=3.95$; $\mathrm{SD}=1.58$ vs. $\mathrm{M}=4.75 ; \mathrm{SD}=1.67 ; \mathrm{t}(76)=-4.39 ; \mathrm{p}<0.0005]$. En el pretest, hay una disparidad de opiniones. Mientras que un $15 \%$ de los estudiantes consideran que no es ético conservar los datos y que las respuestas deben destruirse al momento. En cambio, un 35\% considera ético que se guarden por un tiempo determinado justificando que es posible rendir cuentas con ellos o utilizarlos para nuevos estudios, siempre y cuando se guarde la confidencialidad de los participantes. A su vez, un $20 \%$ considera que primero hay que pedir un permiso para guardarlos Esta obra se comparte bajo la licencia Creative Common Atribución-No Comercial 4.0 International (CC BY-NC 4.0) 
y un $21,25 \%$ piensa que es bueno compartirlos. Finalmente, un $5 \%$ se pregunta si hay alguna normativa al respeto y el 1,25\% considera que al ser una decisión tomada de manera consensuada con el grupo de trabajo es ética. En ningún caso identifican cual es el protocolo que se debe seguir en este tema. En el postest, aunque sigue habiendo comentarios dispares, el 42,5\% de los estudiantes saben cuál es el protocolo que deben seguir y afirman que es necesario conservar la información en un lugar seguro por un tiempo determinado, aunque no reflexionan sobre porqué es necesario. El 11,25\% sigue pensando que se deben destruir, mientras que el $20 \%$ considera que se pueden guardar los datos siempre y cuando se garantice la confidencialidad. El $10 \%$ considera que es necesario pedir el permiso para guardarlos y finalmente un $1,25 \%$ considera que es ético siempre y cuando se llegue a consenso.

\section{Proteger los derechos de los participantes}

\section{Dilema 1: Elección del tema}

Ante el dilema planteado sobre la elección de un tema no discriminatorio, los estudiantes pasan de considerar en el pretest que la respuesta es algo ética $(\mathrm{M}=3.37 ; \mathrm{SD}=1.59)$ a poco ética en el postest $(\mathrm{M}=2.85 ; \mathrm{SD}=1.68)$, siendo la diferencia pre-postest estadísticamente significativa $[\mathrm{t}(72)=2.65 ; \mathrm{p}<0.05]$. En el pretest, un 33,75\% de los estudiantes, realizan una justificación técnica del tema propuesto en el dilema, dando por hecho que como es un tema en el que es necesario investigar, cualquier investigación es válida. Infieren que como la intención no es mala el tema es justificable. Por otro lado, hay un 5\% de estudiantes que determinan que, si existe un consenso en el grupo, el problema ético desaparece, aunque la mayoría de los estudiantes (38,75\%) lo consideran no ético, apelando al criterio de equidad y justicia, ya que el tema que se plantea en el dilema implica una desigualdad entre dos colectivos. Identifican las consecuencias de estigmatización de un colectivo vulnerable, se cuestionan la utilidad de comparar dos grupos que no parten del mismo lugar, apelando a las presunciones y prejuicios que se hacen para establecer ese tema. En el postest se mantienen estos discursos, pero los comentarios en los que se cuestiona el carácter ético de la actuación se convierten en mayoría, aumentando hasta el $60 \%$ de los casos. Identifican en mayor cantidad la desigualdad y la victimización que presenta la elección del tema de estudio propuesto, y también las consecuencias que puede acarrear. En este sentido, en el postest también disminuyen en gran medida los comentarios tecnicistas, pasando a estar presentes en un $21,25 \%$ de los casos y se observa cómo los estudiantes empatizan más con el colectivo, teniendo en cuenta sus emociones y cómo les puede llegar a afectar el estudio. Aun así, algunos estudiantes siguen apelando a la justificación técnica de la necesidad de saber más sobre este ámbito de investigación e intervención. Finalmente, el 6,25\% relacionan la ética al trato directo con los participantes y no a las implicaciones éticas de la elección del tema, como por ejemplo cuando consideran que utilizando un lenguaje respetuoso es suficiente. Por último, la justificación a través del consenso entre el grupo desaparece totalmente.

\section{Dilema 4: Acceso a la muestra}


La formación del compromiso ético en investigación educativa: resultados de una experiencia de innovación docente en educación superior.

Ante el dilema planteado sobre el acceso a la muestra a partir de una persona intermediaria, los estudiantes consideran de media tanto en el pretest como en el postest que la respuesta es poco ética y no existen diferencias significativas entre las dos pruebas. En el pretest, la mayoría de los comentarios -un 60\%- identifican su decisión como una falta de responsabilidad y profesionalidad por parte del equipo, pero no lo consideran una cuestión ética, sino organizativa y técnica. En cambio, un 32,5\% de los casos, cree que el procedimiento es correcto siempre que los datos no sean manipulados por terceras personas y se asegure que la información recogida es veraz, aunque afirman que lo ideal es que fueran personalmente a dar información del estudio y resolver dudas. A su vez, consideran que la persona intermediaria es un recurso aceptable y un buen medio para conseguir la información de los participantes. Solo en un 6,25\% de los casos, se hace referencia a la necesidad de pedir el consentimiento a las personas participantes. Por otro lado, en el postest, se sigue considerando en un $41.25 \%$ de los casos la respuesta del equipo como una falta de implicación y de control del proceso que puede afectar a la veracidad de los datos. Se reduce hasta llegar al 18,75\% de los casos aquellos comentarios que consideran el procedimiento como correcto. Sin embargo, a diferencia del pretest, en el postest, en un 37,5\% de los casos, sí identifican criterios éticos importantes en el momento de juzgar la decisión como, por ejemplo, la necesidad de garantizar la confidencialidad y la protección de los datos, la necesidad de ofrecer un feedback, de pedir permiso al centro y a los participantes y tutores legales antes de la aplicación del instrumento por parte de la persona intermediaria.

\section{Dilema 6: Retorno a los participantes}

Ante el dilema planteado sobre el retorno de los resultados de la investigación a los participantes, los estudiantes consideran de media en el pretest que la actuación es medianamente ética y después de la intervención consideran que es bastante ética, siendo esta diferencia estadísticamente significativa $[\mathrm{M}=4.78 ; \mathrm{SD}=1.28$ vs. $\mathrm{M}=5.34 ; \mathrm{SD}=1.07 ; \mathrm{t}(78)=-3.36$; $\mathrm{p}<0.005]$.

El 73,75\% de los estudiantes consideran en el pretest que la decisión es ética, ya que es necesaria para que las personas encuestadas se sientan partícipes y sepan los resultados antes de publicarlos en otro lugar. Lo ven como un acto de agradecimiento, una ayuda que contribuye al cambio en el centro y en la sociedad, y también como una muestra de implicación y honestidad. Van más allá y, en algunos casos (un 11,25\%) incluso se cuestionan como debe ser este retorno y creen que se debe emplear una comunicación positiva y asertiva. Por otro lado, el 10\% de los estudiantes llegan a cuestionar cómo los resultados pueden afectar a los participantes y plantean la necesidad de mantener el anonimato. En el postest, en el $80 \%$ de los casos les parece una decisión ética, honesta y necesaria, llegando a caracterizarlo como un deber del investigador para con las personas participantes y como una oportunidad de contraste de las interpretaciones. Ponen aún más énfasis en la necesidad de cuidar cómo va a hacerse esta devolución (un 15\% de los casos), y en cómo puede afectar a los participantes del estudio (un 3,75\%.) En este caso, los discursos son más categóricos y no tan reflexivos, ya que se cuestionan en menor medida cómo debe ser este retorno y cómo va a afectar a las personas participantes del estudio. 


\section{Garantizar la propiedad intelectual}

\section{Dilema 3: Citación de las fuentes}

Ante el dilema planteado sobre la propiedad intelectual y el hecho de no citar y referenciar correctamente, existen diferencias estadísticamente significativas en las dos pruebas. En el prestest, los estudiantes consideran que la respuesta a este dilema nada ética y lo reafirman, de manera más contundente, en el postest $[\mathrm{M}=1.43 ; \mathrm{SD}=0.74$ vs. $\mathrm{M}=1.05 ; \mathrm{SD}=0.27 ; \mathrm{t}(79)=$ 4.36; $\mathrm{p}<0.0005]$.

Desde el inicio, en el pretest, el 96,25\% de los encuestados observa la asunción de la poca ética que implica el plagio y saben identificarlo. Tienen un discurso normativo, lo identifican como una cuestión ilegal e inmoral a la par que reconocen la importancia de citar las fuentes, como una cuestión de respeto y de dar crédito al autor. En casos aislados (un 2,5\%), algún estudiante reconoce la ilegalidad, pero le quita importancia y otro lo tilda de comprensible. En el postest, la totalidad de los encuestados se reafirma en la poca ética de esta práctica e incluso desaparecen los comentarios que justifican o encuentran comprensible dicha práctica y buscan alternativas para solucionarlo.

\section{Dilema 7: Autoplagio}

Ante el dilema planteado sobre el autoplagio, los estudiantes consideran de media, tanto en el pretest como en el postest, que la respuesta es algo ética y no hay diferencias estadísticamente significativas entre las dos pruebas. Los estudiantes generalmente consideran que es una decisión ética si se cumplen ciertas condiciones. En el pretest, el 56,25\% consideran que está bien utilizar un trabajo previo para realizar uno nuevo, aunque la mayoría opina que deben pedir permiso al resto del grupo. El 21,25\% de los estudiantes consideran que no es ético copiarlo entero y el 22,5\% indican que deben citar el trabajo anterior. En cambio, en el postest, el $20 \%$ de los estudiantes llegan a identificar la decisión de utilizar el trabajo como autoplagio. El $45 \%$ identifican la necesidad de citar la fuente del trabajo original, además de informar al resto del grupo del uso que se está haciendo de su producto. Sigue habiendo un porcentaje considerable (un 31,25\% de los estudiantes), que solo ve necesario pedir permiso sin verle ningún otro problema, y un 3,75\% que no lo consideran una práctica poco ética si no se copia el trabajo entero.

\section{Discusión y conclusiones}

El estudio pone de manifiesto que el proceso de innovación docente llevado a cabo ha contribuido a desarrollar la competencia ética en la investigación educativa del estudiantado en cinco de los ocho dilemas planteados, teniendo en cuenta las significaciones obtenidas. 
La formación del compromiso ético en investigación educativa: resultados de una experiencia de innovación docente en educación superior.

Los resultados cuantitativos muestran que existen diferencias estadísticamente significativas entre el pretest y el postest en aquellos dilemas éticos de los cuales existen normativas y/o protocolos que indican de qué manera deben proceder las personas investigadoras, como es el caso de la omisión y falsificación de los datos, la citación de las fuentes, la conservación de los datos y el retorno a los participantes (dilemas 3, 5, 6 y 8). Esto se explica por dos motivos. En primer lugar, sucede en los dilemas en que su solución apela al sentido común y a la normativa académica de los estudios superiores, como es el caso la falsificación de los datos y la citación de las fuentes (dilemas 3 y 5). En segundo lugar, en aquellos en los que se ha puesto mayor intensidad pedagógica durante la intervención, ya que se ha hablado específicamente de ellos en los seminarios y se han presentado las normativas y protocolos que guían la actuación en estos casos (dilemas 1, 6,8).

Por otro lado, el hecho que no existan diferencias estadísticamente significativas en el pretest en función de si los participantes han tenido contacto previo con la investigación pone en evidencia que, o bien, la ética en la investigación educativa no se ha abordado en sus experiencias previas, o que los participantes no han desarrollado dicha competencia. Aunque sería necesario un estudio específico sobre el tema para dar una respuesta clara, este dato sirve como comprobación de que parte del estudiantado no estaba en una situación ventajosa sobre el resto y que, por tanto, el conocimiento previo no juega como variable de confusión en este caso.

Por otro lado, los resultados cualitativos indican que ha habido una evolución en todos los dilemas, incluso en aquellos en los que no se perciben diferencias estadísticamente significativas. A nivel general, del pretest al postest, aumenta el porcentaje de estudiantes que identifican adecuadamente la problemática (en todos los dilemas) y disminuyen aquellos discursos que justifican la acción éticamente cuestionable o que se muestran tolerantes con ciertas malas prácticas (dilemas 1, 2, 3, 4, 5, 7 y 8). Además, se puede observar en las respuestas del postest como incorporan en su discurso un lenguaje específico de la formación en ética que no aparecía en el pretest, como cuando citan el consentimiento informado, el anonimato, el retorno a los participantes, el plagio y el autoplagio, etc. Todos estos aspectos son un buen síntoma de que sí que se ha producido un aprendizaje en relación con los criterios éticos básicos incorporados en el currículum de la asignatura en todos los dilemas, a pesar del resultado obtenido a nivel cuantitativo respecto a las medias y las significaciones.

Más allá de las diferencias de contenido en el pretest y el postest, a la hora de realizar el análisis cualitativo del discurso, se observan algunos hechos remarcables. En primer lugar, en el pretest, las personas participantes tienen más integradas las cuestiones éticas que están relacionadas con el marco normativo y los derechos de los participantes y la relación directa con ellos. En cambio, con la incorporación de los contenidos de la asignatura se ha podido observar cómo se amplía el conocimiento sobre otras cuestiones éticas que abarcan todo el proceso de investigación. Esta tendencia a priorizar los estándares éticos relacionados con los derechos de los participantes y la capacidad de integrar a través de la formación elementos clave específicos de la ética en la investigación educativa, va en consonancia con los hallazgos presentados por Hirsch y Esta obra se comparte bajo la licencia Creative Common Atribución-No Comercial 4.0 International (CC BY-NC 4.0) Revista de la Universidad Internacional del Ecuador. URL: https://www.uide.edu.ec/ 
Navia (2018). Ha sido útil para remarcar que la ética es importante durante todo el proceso de investigación y no solo se basa en un código ético (Bridges, 2018).

En segundo lugar, en el pretest, relacionan el carácter ético de sus respuestas con aspectos técnicos, organizativos o de consenso con el grupo. Es decir, si la decisión permite desarrollar con éxito la investigación y/o todos los miembros del grupo están de acuerdo, se considera éticamente aceptable. Estos discursos aparecen sobre todo en aquellos dilemas donde el estudiantado tiene menos conocimientos y recursos éticos para afrontar el conflicto, por lo que recurren a sus conocimientos técnicos como profesionales o a la apelación de la razón consensuada como herramienta de legitimación final. El hecho que en el postest tengan menor peso estos discursos denota que en cierta medida se ha formado un sentido de responsabilidad individual.

En tercer lugar, se observa como en el postest, una vez se conocen las normativas y protocolos que acompañan las decisiones éticas, se da paso a un razonamiento ético de carácter generalmente normativo y en algunos casos se evidencia una falta de reflexión crítica que dificulta la asunción de un comportamiento ético (Colina, 2011; Díaz-Barriga et al., 2016). Aunque el resultado obtenido en estos dos últimos puntos puede evidenciar que se han integrado los aprendizajes esperados y que los contenidos presentados se utilizan de manera adecuada en su discurso, es necesario entender el peligro que se esconde detrás de la legitimación legal del discurso y la opción del consenso como único criterio ético para tomar decisiones de las prácticas, ya que puede llevar al investigador a un estado de conformismo que puede resultar en una investigación aceptada y legal, pero no éticamente responsable (Zgaga, 2020).

Según Espinoza y Calva (2020) trabajar la competencia ética en investigación no solo conduce a unos resultados más fiables en los estudios realizados, sino que impacta en la persona que investiga, por lo que promover el desarrollo de esta competencia en el aula no solo servirá para mejorar la calidad de la investigación, sino que promoverá en la persona un sentido de responsabilidad a nivel personal y profesional. A través de este estudio, hemos podido comprobar que es posible enseñar y evaluar la competencia ética en el estudiantado dentro de la educación superior y específicamente en los primeros grados de consecución tal como Dreyfus y Dreyfus (2004) exponen. Aun así, consideramos que el trabajo realizado es un buen punto de partida para iniciar la reflexión y la práctica en la asignatura de investigación educativa desde una perspectiva ética, pero es posible incorporar nuevos recursos prácticos que permitan desarrollar aquellos aspectos de la competencia que han obtenido peores resultados en la innovación introducida. Por ejemplo, el marco de evaluación ética CERD (consecuencialista, ecológico, relacional y deontológico) propuesto por Stutchbury y Fox (2009) podría ser útil para profundizar en los procesos reflexivos de la dimensión ética de la investigación educativa desde una perspectiva práctica (Fox et al., 2020; Fox y Mitchell, 2019), que sería útil para superar la visión normativa que han presentado los estudiantes encuestados, fomentar la reflexión crítica e introducir nuevos contenidos curriculares. 
Más allá de los resultados obtenidos en la aplicación del instrumento, se hace necesario destacar las oportunidades y limitaciones que este nos brinda. En primer lugar, aunque no podemos afirmar que sea una herramienta que desarrolle la formación del compromiso ético en sí misma, sí que contribuye a su desarrollo en tanto que estimulan los procesos reflexivos en el alumnado y son útiles para que el profesorado pueda revisar la intencionalidad de su intervención educativa. En referencia con lo expuesto, la innovación presentada da respuesta a la importancia de disponer de instrumentos y herramientas que permitan diseñar una intervención efectiva, además de dar a las asignaturas un enfoque más competencial, permitiendo recoger evidencias y evaluar los progresos del estudiantado. Asimismo, también permiten trabajar a partir de evidencias y de desarrollar tecnología educativa para poder sistematizar las mejoras introducidas en el aula, evaluarlas y potenciar el desarrollo de competencias del estudiantado. Finalmente, cabe destacar la posibilidad que ofrece este instrumento de ser transferido o aplicado en otras asignaturas o cursos que incluyan procesos de investigación, de evaluación, de detección de necesidades, etc. como, por ejemplo, los trabajos de fin de estudios o las prácticas profesionales.

A modo de conclusión, se puede afirmar que el desarrollo de la competencia ética requiere voluntad e intención por parte del profesorado para repensar sus prácticas e introducir cambios enfocados a la dimensión ética en sus asignaturas, pero este esfuerzo no será suficiente sin el compromiso de las instituciones educativas y del estudiantado por convertir la responsabilidad ética en una competencia general de la educación superior.

\section{Referencias bibliográficas}

Agència per a la Qualitat del Sistema Universitari de Catalunya (2014). Universitat i treball a Catalunya 2014: Estudi de la inserció laboral de la població titulada de les universitats catalanes. https://www.aqu.cat/doc/doc_14857668_1.pdf

American Psychological Association (2017). Ethical Principles of Psychologists and Code of Conduct with the amendment to Standard 3.04. American Psychological Association. https://www.apa.org/ethics/code/ethics-code-2017.pdf

Artino, A. R. (2007). Assessing Ethical Dilemmas in Educational Research: Does Formal Ethics Training Make a Difference? Journal of College and Character, 8(5). https://doi.org/10.2202/1940-1639.1616

Bridges, D. (2018). Epistemology, Ethics and Educational Research en P. Smeyers \& M. Depaepe (Eds.), Educational Research: Ethics, Social Justice, and Funding Dynamics, (pp. 109120). Springer. https://doi.org/10.1007/978-3-319-73921-2_8

Brown, D. (2019). Ethics. En C. Opie \& D. Brown (Eds.), Getting Started in your Educational Research (pp. 76-94). SAGE Publications.

Buendía, L., \& Berrocal, E. (2001). La Ética de la Investigación Educativa. Ágora digital, 1(9). http://hdl.handle.net/10272/6606

Canimas, J. (2019). Sobre la distinción entre problemáticas éticas, tecnocientíficas y políticas en M.J. Úriz (Coord.), La necesaria mirada ética en Trabajo Social (pp. 11-22). Ediciones Paraninfo y Consejo General del Trabajo Social.

Esta obra se comparte bajo la licencia Creative Common Atribución-No Comercial 4.0 International (CC BY-NC 4.0)

Revista de la Universidad Internacional del Ecuador. URL: https://www.uide.edu.ec/ 
Chávez, G., y Benavides, B. (2011). Los profesores universitarios: entre la exigencia profesional y el compromiso ético-social. Sinéctica. Revista Electrónica de Educación., 37, 1-13. http://www.scielo.org.mx/scielo.php?script=sci_arttext\&pid=S1665109X2011000200003

Colina, A. (2011). La ética profesional y el narcisismo benigno en la formación de la identidad del investigador en educación en México. Revista Iberoamericana De Educación Superior, (2)4, 135-148. https://doi.org/10.22201/iisue.20072872e.2011.4.41

de Jager, T. (2012). Can First Year Student's Critical Thinking Skills Develop in a Space of Three Months? Procedia - Social and Behavioral Sciences, 47, 1374-1381. https://doi.org/10.1016/j.sbspro.2012.06.829

Díaz-Barriga, F., Pérez-Rendón, M., y Lara-Gutiérrez, Y. (2016). Para enseñar ética profesional no basta con una asignatura: los estudiantes de Psicología reportan incidentes críticos en aulas y escenarios reales. Revista Iberoamericana De Educación Superior, 7(18), 42-58. https://doi.org/10.22201/iisue.20072872e.2016.18.175

Dreyfus, H. L., \& Dreyfus, S. E. (2004). The ethical implications of the five-stage skill-acquisition model. Bulletin of Science, Technology and Society, 24(3), 251-264. https://doi.org/10.1177/0270467604265023

Espinoza, E. E., y Calva, D. X. (2020). La ética en las investigaciones educativas. Revista Universidad y Sociedad, 12(4), 333-340. https://bit.ly/2Wz2Ym1

Fox, A., Baker, S., Charitonos, K., Jack, V., \& Moser-Mercer, B. (2020). Ethics-in-practice in fragile contexts: Research in education for displaced persons, refugees and asylum seekers. British Educational Research Journal, 46(4), 829-847. https://doi.org/10.1002/berj.3618

Fox, A., \& Mitchell, R. (2019). Ethical learning from an educational ethnography: the application of an ethical framework in doctoral supervision en H. Busher \& A. Fox (Eds.), Implementing Ethics in Educational Ethnography: Regulation and Practice (pp. 110-126). Routledge.

García, R. (2006). El profesorado universitario ante la ética profesional docente. Revista Española de Pedagogía, 64(235), 545-566. https://bit.ly/3zT9mTv

González, V. (2004). El profesorado universitario: su concepción y formación como modelo de actuación ética y profesional. Revista Iberoamericana de Educación, 34(1), 1-11. https://doi.org/10.35362/rie3412934

Healey, R. L. (2014). How engaged are undergraduate students in ethics and ethical thinking? An analysis of the ethical development of undergraduates by discipline. Student Engagement and Experience Journal, 3(2). https://doi.org/10.7190/seej.v3i2.93

Hirsch, A., y Navia, C. (2018). Ética de la investigación y formadores de docentes. Revista Electrónica de Investigación Educativa, 20(3), 1-10. https://doi.org/10.24320/redie.2018.20.3.1776

López, R. (2013). Ética profesional en la formación universitaria. Perfiles educativos, 35(142), 15 24. https://bit.ly/2V9hJf5

McLeod-Sordjan, R. (2014). Evaluating moral reasoning in nursing education. Nursing Ethics, 21(4), 473-483. https://doi.org/10.1177/0969733013505309

Esta obra se comparte bajo la licencia Creative Common Atribución-No Comercial 4.0 International (CC BY-NC 4.0)

Revista de la Universidad Internacional del Ecuador. URL: https://www.uide.edu.ec/ 
Opazo, H. (2011). Ética en investigación: Desde los Códigos de Conducta hacia la Formación del Sentido Ético. REICE. Revista Iberoamericana sobre Calidad, Eficacia y Cambio en Educación, 9(8), 61-78. https://www.redalyc.org/pdf/551/55119127005.pdf

Ortega, P. (2020). Educar desde la experiencia ética del otro. Revista Boletín Redipe, 9(2), 23-32. https://doi.org/10.36260/rbr.v9i2.908

Paz, E. J. (2018). La ética en la investigación educativa. Revista Ciencias Pedagógicas e Innovación, 6(1), 45-51. https://doi.org/10.26423/rcpi.v6i1.219

Raykov, M. (2020). Education researchers' perceptions of and experiences with the research ethics application process in Europe and beyond. European Educational Research Journal, 19(1), 10-29. https://doi.org/10.1177/1474904119893461

Riberas, G., Rosa, G., y Vilar, J. (2014). Nuevas metodologías para la formación de profesionales reflexivos en educación social. Revista CIDUI, 2, 1-14. https://www.cidui.org/revistacidui/index.php/cidui/article/view/630/606

Sánchez, C. (1997). Dilemas éticos de la investigación educativa. Revista de Educación, 312, 271 280. https://sede.educacion.gob.es/publiventa/detalle.action?cod=460

Stutchbury, K., \& Fox, A. (2009). Ethics in educational research: Introducing a methodological tool for effective ethical analysis. Cambridge Journal of Education, 39(4), 489-504. https://doi.org/10.1080/03057640903354396

Tey, A., Vilà, R., y Martínez, M. (2014). Competencias para el aprendizaje ético en estudiantes universitarios de enfermería y pedagogía. REDU. Revista de Docencia Universitaria, 12(1), 337-352. https://doi.org/10.4995/redu.2014.6422

Zgaga, P. (2020). Researchers and research ethics: Between fears of the expansion of controversial practices and the strengthening of ethical awareness. European Educational Research Journal, 19(1), 43-55. https://doi.org/10.1177/1474904118788487 


\section{Apéndice}

\section{Instrumento: Dilemas éticos aplicados a la investigación educativa}

Estimado/a, A continuación, te presentamos una serie de situaciones frecuentes que se pueden dar cuando llevamos a cabo un trabajo de investigación. Te pedimos que las puedas leer atentamente y contestes, con la máxima sinceridad, en una escala del 1 al 6 , la respuesta que más concuerda con tu punto de vista. Recuerda que no es un examen y que no hay preguntas buenas ni malas; las respuestas sólo recogen diferentes estilos de pensar y de actuar. Estas situaciones, te permitirán reflexionar sobre cómo posicionarte ante determinadas decisiones a tomar durante el proceso de elaboración de un trabajo de investigación. El tiempo estimado para contestar es de 20 minutos. Muchas gracias.

\section{Introducción}

María, Joel, Paula, Martín y Carla son un grupo de estudiantes universitarios que tienen que realizar una investigación en el ámbito socioeducativo. Durante este proceso deberán tomar una seria de decisiones relacionadas con el diseño del estudio. A continuación, se presentan algunas de las situaciones con las que se encuentran durante el proceso de elaboración del trabajo de investigación y las decisiones que han tomado.

Lee cada situación, y marca la casilla correspondiente en función de si la decisión que han tomado te parece "Nada ética" (1) o "Muy ética" (6). A continuación, justifica de manera concisa tu respuesta.

\section{Situación 1}

Uno de los primeros pasos a realizar es escoger el tema de investigación. Varios integrantes del grupo están muy interesados en el ámbito de la diversidad funcional.

María, una de las componentes, propone realizar una investigación comparando las capacidades sociolaborales de una persona sin discapacidad y otra que sufre una afectación cognitiva moderada.

Diría que la propuesta de tema que hace María es...

\begin{tabular}{llllll}
\hline 1 & 2 & 3 & 4 & 5 & 6 \\
Nada ética & Poco ética & Algo ética & Medianamente ética & Bastante ética & Muy ética
\end{tabular}

Justifica tu respuesta:

\section{Situación 2}

Joel, otro miembro del grupo, propone recoger la información a partir de un cuestionario en formato de encuesta, ya que es un método que ha utilizado antes y lo domina. Paula cree que sería mejor recoger la información de otra manera, pero no sabe cómo, ya que dice que con el 
La formación del compromiso ético en investigación educativa: resultados de una experiencia de innovación docente en educación superior.

cuestionario hay información que no podrán recoger en profundidad. Finalmente, el grupo lo discute y toma la decisión de utilizar la encuesta y aprovechar así la experiencia de Joel.

Diría que la decisión del grupo de utilizar un cuestionario es...

\begin{tabular}{llllll}
\hline 1 & 2 & 3 & 4 & 5 & 6 \\
Nada ética & Poco ética & Algo ética & Medianamente ética & Bastante ética & Muy ética \\
\hline
\end{tabular}

Justifica tu respuesta:

Situación 3

El grupo se pone a hacer el trabajo y buscan información en internet. Martín encuentra un trabajo de un grupo de estudiantes de otra universidad muy parecido al suyo. Al revisarlo, lee una definición muy interesante que cree que tiene que estar en su trabajo, así que la incorpora al marco teórico, pero no cita la fuente porque no encuentra el nombre del autor.

Diría que la decisión de Martín de incorporar la definición es...

\begin{tabular}{llllll}
\hline 1 & 2 & 3 & 4 & 5 & 6 \\
Nada ética & Poco ética & Algo ética & Medianamente ética & Bastante ética & Muy ética \\
\hline
\end{tabular}

Justifica tu respuesta:

Situación 4

Llegó el momento de pasar la encuesta. Carla dice que su madre, que es educadora social, puede pasar las encuestas a los chicos y chicas con discapacidad del Centro Especial de Trabajo donde ella trabaja. Al grupo le parece bien, le envían directamente por correo electrónico la encuesta a la madre de Carla y ella le devuelve todas las encuestas rellenadas al cabo de dos semanas.

Diría que el procedimiento de recogida de datos es...

\begin{tabular}{llllll}
\hline 1 & 2 & 3 & 4 & 5 & 6 \\
Nada ético & Poco ético & Algo ético & Medianamente ético & Bastante ético & Muy ético \\
\hline
\end{tabular}

Justifica tu respuesta:

Situación 5

El grupo está revisando y analizando los resultados de la encuesta. Se encuentran que una de las encuestas contiene una pregunta que no está contestada. Martín y María deciden contestarla y poner un 3, la puntuación media de la escala de respuesta. De esta manera pueden utilizar la encuesta porque si no la deberían descartar y no llegarían al número mínimo que se les pide para hacer el trabajo.

Diría que la decisión de Martín y María es...

Esta obra se comparte bajo la licencia Creative Common Atribución-No Comercial 4.0 International (CC BY-NC 4.0)

Revista de la Universidad Internacional del Ecuador. URL: https://www.uide.edu.ec/ 


\begin{tabular}{llllll}
\hline 1 & 2 & 3 & 4 & 5 & 6 \\
Nada ética & Poco ética & Algo ética & Medianamente ética & Bastante ética & Muy ética \\
\hline
\end{tabular}

Justifica tu respuesta:

Situación 6

Los resultados de la investigación son muy interesantes, aunque salen datos que quizás no gustan al centro donde se ha pasado el cuestionario. El grupo lo comenta y llegan al acuerdo de ir al centro y explicar los resultados que han obtenido y hacer un retorno de las conclusiones del trabajo a todas las personas implicadas.

Diría que la decisión del grupo es...

\begin{tabular}{llllll}
\hline 1 & 2 & 3 & 4 & 5 & 6 \\
Nada ética & Poco ética & Algo ética & Medianamente ética & Bastante ética & Muy ética \\
\hline
\end{tabular}

Justifica tu respuesta:

Situación 7

El trabajo ha quedado muy bien y les han puesto muy buena nota. La María, que era quien propuso el tema, está especialmente contenta y decide continuar con la misma investigación con su TFG. De este modo, aprovecha algunas de las partes del trabajo que ella hizo y las incorpora al TFG, ampliando un poco otros apartados.

Diría que la decisión de María es...

\begin{tabular}{llllll}
\hline $\mathbf{1}$ & $\mathbf{2}$ & $\mathbf{3}$ & $\mathbf{4}$ & $\mathbf{5}$ & $\mathbf{6}$ \\
Nada ética & Poco ética & Algo ética & Medianamente ética & Bastante ética & Muy ética \\
\hline
\end{tabular}

Justifica tu respuesta:

Situación 8

Una vez finalizado el trabajo, los miembros del grupo se preguntan qué hacer con los cuestionarios y los datos originales almacenados electrónicamente. Joel propone destruirlos para proteger la confidencialidad de las personas que los contestaron. Tras discutirlo con el grupo, finalmente deciden eliminar los datos de la carpeta compartida del drive y guardarlos en una carpeta con contraseña en el disco duro del ordenador de Paula y conservarlos durante un tiempo prudencial.

Diría que la decisión del grupo es...

\begin{tabular}{llllll}
\hline 1 & 2 & 3 & 4 & 5 & 6 \\
Nada ética & Poco ética & Algo ética & Medianamente ética & Bastante ética & Muy ética \\
\hline
\end{tabular}
Justifica tu respuesta: 